\title{
A new complexity reduction methods of V-BLAST MIMO system in a communication channel
}

\author{
Sunita Panda ${ }^{1}$, Rosalin Samantaray ${ }^{2}$, Pradyumna Ku. Mohapatra ${ }^{3}$, R.N.Panda ${ }^{4}$, Padma Sahu $^{5}$ \\ ${ }^{1}$ Department of Electronics \& Communication Engg, GITAM. University, India \\ ${ }^{2}$ Department of Computer Science \& Engg, BEC, Odisha, India \\ ${ }^{3}$ Department of Electronics \& Communication Engg, OEC Odisha, India \\ ${ }^{4}$ Department of Electronics \& Communication Engg, GIFT Odisha, India \\ ${ }^{5}$ Department of Electronics \& Communication Engg, KIT Odisha, India
}

\section{Article Info}

Article history:

Received Oct 2, 2018

Revised Dec 20, 2018

Accepted Jan 15, 2019

Keywords:

MIMO-OFDM

MMSE

QR decomposition

VBLAST

$\mathrm{ZF}$

\begin{abstract}
To design most reliable wireless communication system we need an efficient method which can be proposed in this paper is V-BLAST technique which is most powerful tool in MIMO system. To improve the channel capacity and data rate efficiently we need manifold antennas together with the transmitter and receiver. In this paper we have analysed different equalizers performance using V-BLAST algorithm. We have proposed two methods i.e low complexity $\mathrm{QR}$ algorithm and another is consecutive iterations reduction method. This methods compare with traditional finding methods such as ZF, MMSE, SIC and ML. The proposed algorithm shows that it not only reduce the computational complexity but we can achieve significant bit error rate (BER) and probability error compared to traditional VBLAST techniques.
\end{abstract}

Copyright () 2019 Institute of Advanced Engineering and Science. All rights reserved.

\section{Corresponding Author:}

Pradyumna Ku. Mohapatra,

Department of Electronics and Communication Engineering,

S.O.A University, Bhubaneswar, Odisha, India.

Email: er_pradyumna@yahoo.co.in

\section{INTRODUCTION}

In high speed communication, MIMO [1] systems are found to be most useful and viable method in wireless technology. Wireless communication is a system in which both transmitting as well as receiving end consists of various antenna rudiments as shown in Figure 1. To improve the data rates in MIMO system [2] we have to design both transmitting as well as receiving antenna in such a amanner that each user in a system will be enhanced.

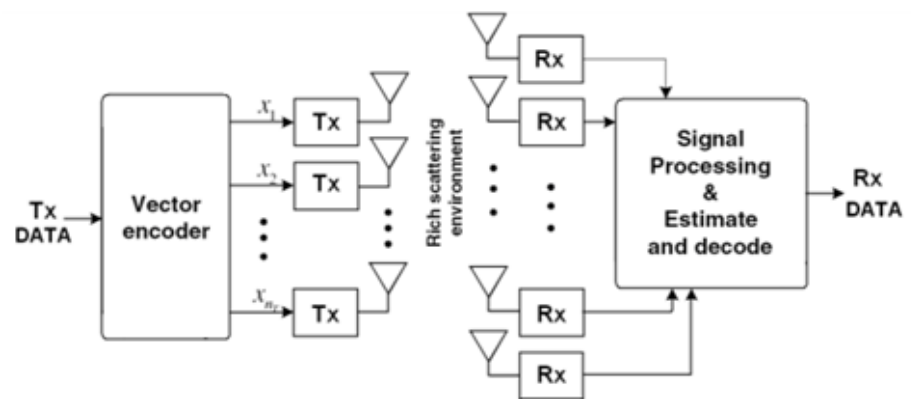

Figure 1. V-Blast system 
The great advantage of MIMO system in wireless channels is that it has the ability of robustness to the cause of multipath fading, besides flexible higher ability, spectral efficiency and improved BER presentation. To enhance system BER and spatial diversity gain which can be achieved if we follow the proper method which can analyze various signals from end to end diverse antennas to clash fading. The presentation of the given system proposed in this paper may analyzed in terms of BER and probability of error which can be strictly affected by fading. To enhance quality of wireless communication system there are different methods adopted. One of the most promising techniques used in MIMO system is V-BLAST which mimics vertical-bell laboratories layered space time. This means it acts on layered space, time coding Recipient side of the system blast is connected. This is too identified as a receiver signal processing algorithm. V-BLAST [3] is used to abridged the perversion, noise because of nosiness from the channel as compared to previous methods. Because of its performance like higher spectral efficiency with high power and functioning complexity is low we will get better result than D-BLAST and almuti techniques. One of the advantages of this technique is that it detects dominant signal or maximum SNR and then it regenerate the arriving signal from the existing channel. Generally low complexity linear MIMO detectors are based on MMSE. Because of inability of traditional detectors particularly in multiple inputs and multiple outputs systems that uses a fewer digits of antenna. Therefore we have to develop the system called V-BLAST algorithm which provides successive interference cancellations in the suitable manner. To accomplish the elevated spectral efficiency with realistic modified difficulty in prosperous dispersal environment in the course of exploiting spatial measurement we need the decoder like VBLAST system with Successive Interference Cancellation (SIC) [4] which results advanced diversity gains and enhance BER presentation.

\section{SYSTEM MODELS}

In MIMO scheme consists of number of transmitters and receivers and we assume channel is flat fading Rayleigh channel. The signal is contaminated by zero mean additive white Gaussian noise (AWGN) [5-6]. The arriving signal be able to describe as:

$$
r=\dot{H} X+n
$$

Now

$$
r=\left[y_{1,} y_{, 2} y_{3}, y_{r}\right]^{T}
$$

And the channel matrix is shown in the given equation

$$
\dot{H}=\left[\begin{array}{cccc}
h_{11} & h_{12} & \ldots & h_{1 N t} \\
h_{21} & h_{22} & \ldots & h_{2 N t} \\
\vdots & \vdots & \ddots & \vdots \\
h_{N r 1} & h_{N r 2} & \ldots & h_{N r N t}
\end{array}\right]
$$

Where, $\dot{H}$ is a channel impulse response, transmitted signal

$$
X=\left[x_{1}, x_{2} x_{N t}\right]^{T}
$$

And noise

$$
n=\left[n_{1}, n_{2} n_{N r}\right]^{T}
$$

\section{SIMULATIONS OF MIMO CHANNEL CAPACITY}

In multiple input and multiple output system having $M_{-} T$ transmit antennas and N_R receiving antennas and the channel transfer matrix is represented as $\mathrm{H}$ with dimension $\mathrm{M} \_\mathrm{T} \times \mathrm{N} \_\mathrm{R}$.The output signal can be described as in equation (1). We presume a MIMO downlink with M_T transmit antennas at the base transceiver station and N_R receive antennas at the mobile station, where the channel is recognized to the receiver but not to the transmitter. When the transmitted signals are independent with equal power at each antenna, the ergodic channel capacity taken over the probability distribution of $\mathrm{G}$ is given by:

$$
C=E\left\{\log _{2}\left[\operatorname{det}\left(I_{N R}+G \frac{P}{M T \times N}\right]\right\}\right.
$$


Where $\mathbf{G}$ is an $M_{T} \times N_{R}$ transfer matrix of the flat-fading channel, $P$ is the total transmitted power, and $N$ is the variance of the independent Gaussian noise at each receive antenna.

Channel capacity describes utmost error-free data rate that a channel can hold .In Figure 2 shows MIMO channel capacity for AWGN in a variable transmitting antenna keeping a fixed number of receiving antenna and Figure 3 also identifies how channel capacity affects in variable number of transmitting and receiving antenna.

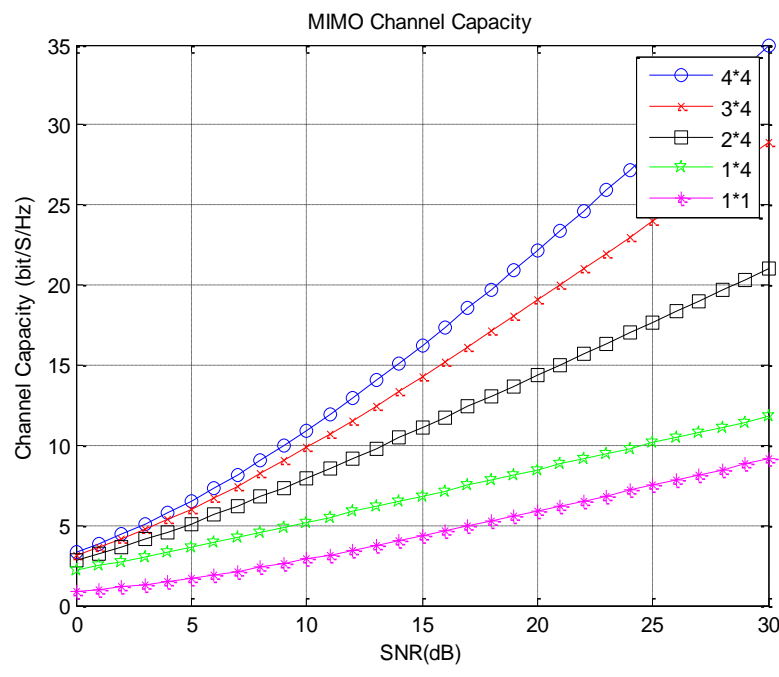

Figure 2. MIMO channel capacity (variable transmitting antenna keeping a fixed number of receiving antenna)

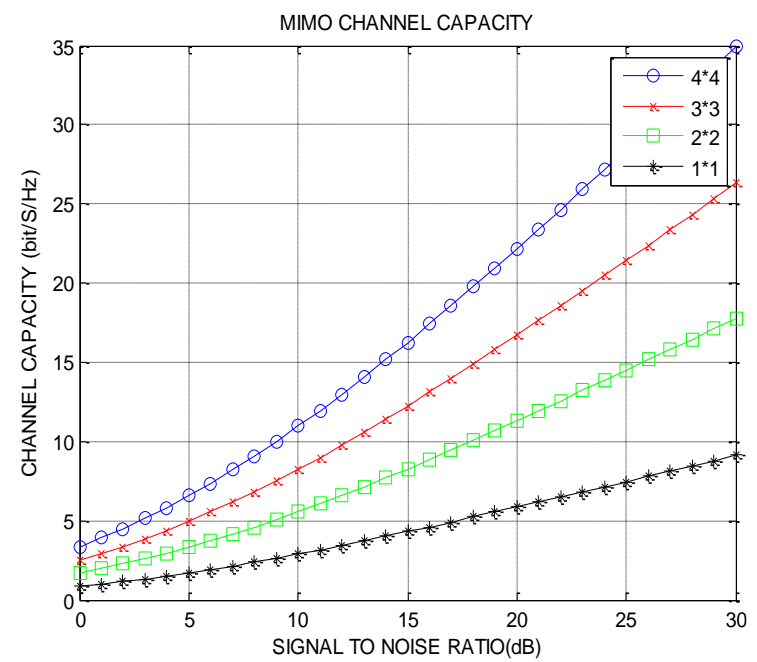

Figure 3. MIMO channel capacity (variable number of transmitting and receiving antenna)

\section{V-BLAST PROCEDURE:}

Generally in transmitting system a information is decoded and addicted to a number of sub-sections called as layers. The layers are precise parallel transversely the freedom and the cycling process is disconnected before communication. Arriving signal at every end of take delivery of antenna is a superposition of $\mathrm{N}$ faded symbols with AWGN. Particularly in BLAST systems, i.e. space and time, identification method is obtained upright for every received vector .Exclusively beating of simplification, suppose that the initial character is to be identified. The identification procedure mainly two steps, i.e., nosiness repression and interference cancellation (subtraction). The containment process nulls out intrusion by analytical the arriving vector onto the unacceptable subspace of the subspace spanned with the inquisitive information. Following that, the usual finding of the primary symbol is done. Intervention termination is nothing but identified symbol is eliminated as of the arriving vector. The BLAST discovery method combines both linear as well as nonlinear algorithms. In a simulation using matlab R2009 simbol error rate of V -BLAST $16 \times 4$ QAM can be measured as shown Figure 4. Starting with generation of QAM signal and then add with AWGN and finally decoding the received symbol for various $\frac{E_{b}}{N_{O}}$ values. 


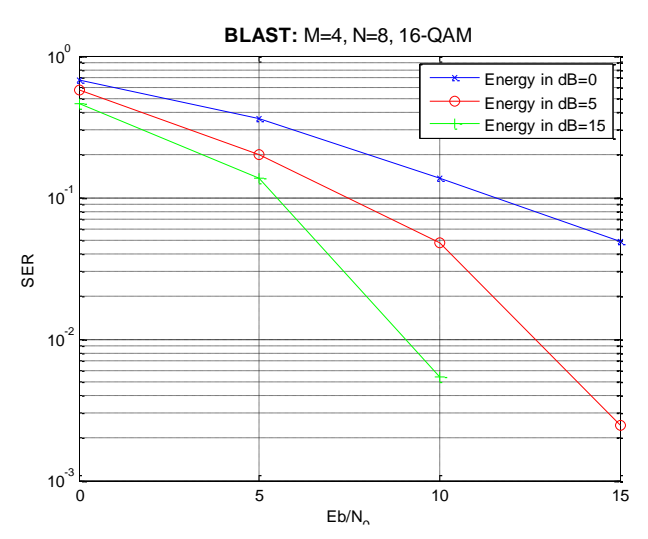

Figure 4. SER performances of V-BLAST $16 \times 4$ QAM

\section{V-BLAST DETECTION METHODS:}

BLAST MIMO detection can be classified in two categories i.e: 1) Linear ( ZF and MMSE), 2) Nonlinear (ML and VBLAST)

Performance of both liner nonlinear detection scheme can be analyzed through an index called as bit error rate (BER). Nonlinear V-BLAST detection algorithm provides a good BER performance compared to liner one.

\subsection{Zero Forcing (ZF)}

It is one of the most suitable and simplest detection scheme in V-BLAST MIMO system. Generally zero forcing is nothing but opposite filter which is to recompense the channel output function. As it is a linear equalizer, it does not take the noise but will perform better in high signal to noise ratio. We consider $Y_{Z F}$ is the zero forcing equalizer matrix then it must assure the following criteria.

$$
Y_{Z F} H=1
$$

Now,

$$
Y_{Z F}=\left(H^{T} \mathrm{H}\right)^{-1} H^{T}
$$

As far as performance evaluation like bit error rate and signal to noise ratio is concerned we may consider ZF with V-BLAST provides better result than usual ZF which has been simulated in Figure 5. In this simulation BER performances of V-BLAST $2 \times 2$ ZF-PIC,ZF-OSIC detector is better than traditional ZF decoder. In Figure 6 shows bit error rate performances of V-BLAST ZF detector keeping fixed receiving antenna.

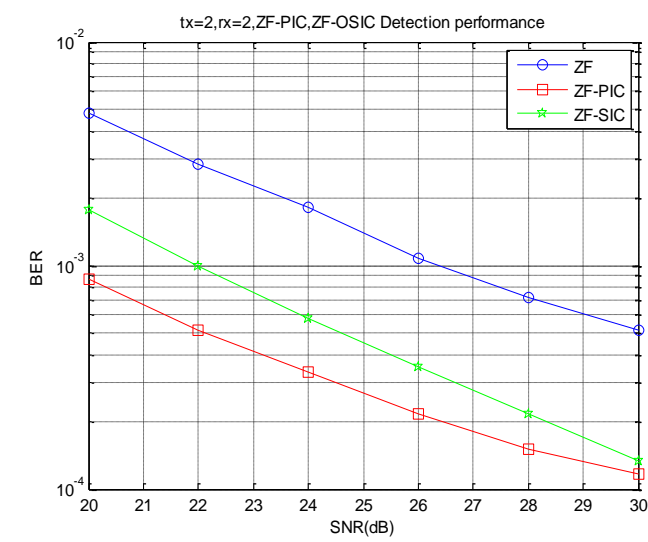

Figure 5. BER performances of V-BLAST $2 \times 2 \mathrm{ZF}-$ PIC.ZF-OSIC detector

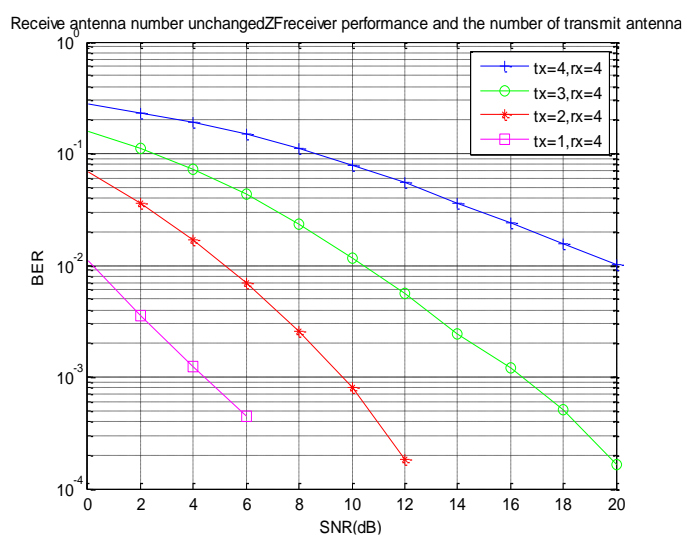

Figure 6. V-BLAST ZF detector (unchanged receiving antenna 


\subsection{Minimum Mean Square Error (MMSE)}

As far as performance is concerned between $\mathrm{ZF}$ and MMSE we will consider MMSE rather than ZF because MMSE discriminate together with intrusion and noise parameters, while the ZF eliminate only the noisy parameters. If we consider mean square error is the main criteria then MMSE receiver is the best choice as far as the linear detector is concerned. If $Y_{M M S E}$ is the MMSE matrix then. If we consider with high SNR, MMSE equalizer works as a zero forcing equalizer.

$$
Y_{M M S E}=\left(H^{T} \mathrm{H}+\frac{1}{\mathrm{SNR}} \mathrm{I}\right)^{-1} H^{T}
$$

\subsection{Successive interference cancellation (SIC)}

Generally the result of the previously identified symbol is used to withdraw the interference of the successive symbol if the symbols are detected successively. It gives rise to a method called the decision directed detection mode and also it is called as Successive Interference Cancellation(SIC) [7].

As far as low complexity is concerned sub-optimal algorithm for ZF-SIC,MMSE-SIC consist of following steps:

a. Ordering: Decide the most favourable detection order corresponds to to locate out the Tx stream with minimum error discrepancy.

b. Interference Cancellation: Approximation the signal from the transmitter is taken as a strong deterministic and the signal stream can be cancel out the weaker signals using the nulling vector $W_{K I}$. So $W Y_{K I}=W_{K I}^{T} r$

c. Slicing: The Approximate word of the useful sending signal is identified with slicing to the adjacent worth in the signal group.

d. Interference Cancellation: Subtracting the input of the identified representation from the residual arriving signal vector and go back to the ordering step.To reduce the detection difficulty, the useful sending signal has been identified and its outcome should be null and void from the inward signal vector for the remaining transmitting signal.

In a simulation graph as shown in Figure 7 which describes error probability of V-BLAST $2 \times 2$ ZF,ZF-OSIC,MMSE AND MMSE-OSIC

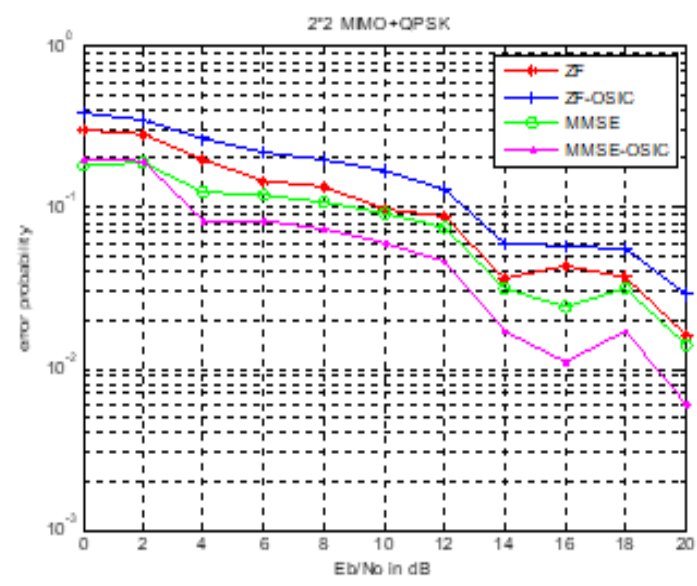

Figure 7. Error probability of V-BLAST $2 \times 2$ ZF, ZF-OSIC, MMSE and MMSE-OSIC

\subsection{MAXIMUM LIKELIHOOD (ML):}

It is one of the equalization method used in non linear channel. Several draw backs which comes in ZF and MMSE and also SIC detection method which can be resolved in this method. Even though computational complexities more than the previous method but presentation of this detection technique is more reliable than previous one. This techniques work on the rule of maximum likelihood [8] between the arriving signal and the multiply of channel matrix $\mathrm{H}$ with the Tx signals to locate out the one with least distance. The approximate transmitted signal vector $\mathrm{X}$ can be found using ML detection method which is in the following equation 


$$
\widehat{X_{M L}}=\underset{\hat{x}}{\operatorname{argmin}\left[|Y-H X|_{F}\right]^{2}}
$$

F can be taken as Frobenius norm. In Figure 8 shows comparison BER graph of $4 \times 4$ MMSE-SIC and MLdetector and Figure 9 which describes how V-BLAST QR decomposition detection better than traditional ZF detector.

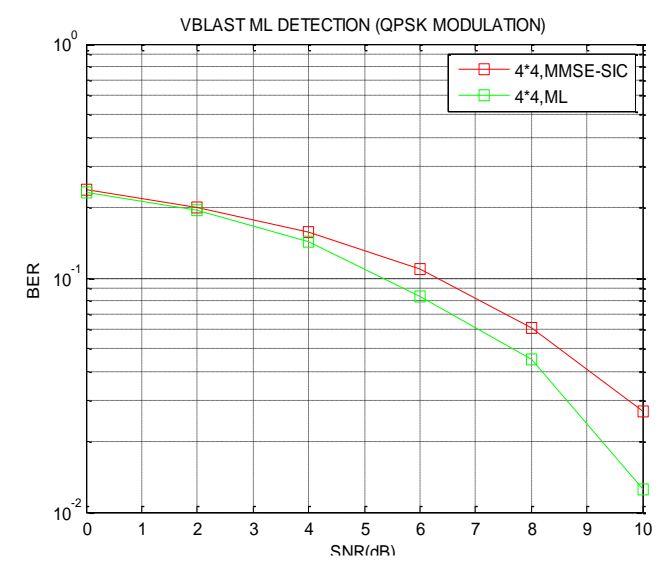

Figure 8. BER performances of V-BLAST ML detection (QPSK)

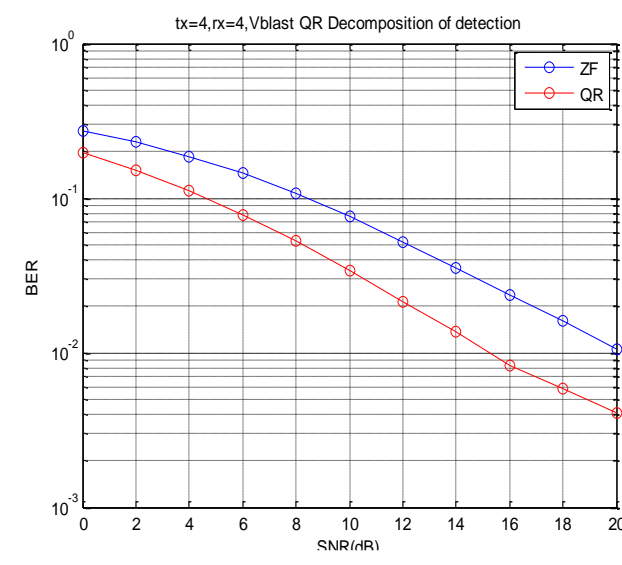

Figure 9. BER performances of V-BLAST QR decomposition detection

\subsection{VBLAST-STBC:}

VBLAST-STBC [9-12] system consist of $M$ transmit and $N$ receive antennas and the transmitted symbols are multiplexed into $(\mathrm{X}+\mathrm{Y})$ layers, the higher $\mathrm{X}$ layers are denoted as the VBLAST layers, in which the symbols are transmitted by the first $\mathrm{X}$ antennas. The the other layers, $(\mathrm{X}+1) \sim \mathrm{C}$, are denoted as the STBC layers, in which the symbols are encoded with $\mathrm{g} 2$ code and are transmitted by $2 \mathrm{C}$ antennas. The channel can be described as $H \in Y^{N-M}$. Where channel state coefficient can be denoted as $h_{i j}$ Which is a complex gain from it transmitted antenna to jth receive antenna. The system function can be represented as $r=\dot{H} X+$ $n$, where $\mathrm{r}$ is the received matrix, and $\mathrm{n}$ is the AWGN noise matrix and $\mathrm{X}$ is the transmitted block matrix. The transmitted power of each symbol in $\mathrm{X}$ is defined as $E\left[\left|x_{i j}\right|^{2}\right]=\frac{E_{x}}{M}$ Where $E_{x}$ is the total transmitted power. At the receiver, the channel matrix $\dot{H}$ is decomposed as $\dot{H}=Q R$ by exploit QR decomposition.

\subsection{Q-R decomposition}

It is one of the methods to solve matrix inversion problem and can be calculated with $\dot{H}=[Q] *[R]$. Figure 9 shows BER performance of V-BLAST QR decomposition detection. Complexity issue: As far as computational complexity is concerned the above decoders mention in this paper with $\mathrm{H}$ is high. In order to improve the computational complexities we have to modify it and set a minimum complexities with $\mathrm{QR}$ decomposition is proposed. The dimensions $4 \mathrm{~N} \times(\mathrm{M}-1)$ are used for departure symbols with its channel matrix $\mathrm{H}$ can be replaced with $\mathrm{N} \times \mathrm{M}$. The complexity reduction with $\mathrm{QR}$ decomposition is invalid for the dimension $\mathrm{N}<\mathrm{M}-1$.

\section{PROPOSED LC-QR ALGORITHM}

Step i. Generally decomposition of $Q R$ with $\dot{H}$ can be performed by the following matrix operation i.e $\dot{H}=Q R$

$$
\left[\begin{array}{cccc}
Q_{11} & Q_{12} & \cdots & Q_{1 M} \\
Q_{21} & Q_{22} & \ldots & Q_{2 M} \\
\vdots & \vdots & \vdots & \vdots \\
Q_{N 1} & Q_{N 2} & \ldots & Q_{N M}
\end{array}\right]\left[\begin{array}{cccc}
R_{11} & R_{12} & \ldots & R_{1 M} \\
0 & R_{22} & \ldots & R_{2 M} \\
0 & 0 & \ldots & \vdots \\
0 & 0 & 0 & R_{M M}
\end{array}\right]
$$

Step ii. $\quad$ If $N \geq M$ go to step-iii else step:vii 
Step iii. Approximate message signal of STBC layer with $2 \times 2$ decoder

Step iv. V-BLAST layer depends on the initial approximate message signal of STBC

Step v. Nosiness deletion or cancellation

Step vi. $\quad$ STBC level with STBC $2 \times \mathrm{N}$ decoder

Step vii. If $\mathrm{N}=\mathrm{M}-1$ go to Step-viii else step-xii (invalid)

Step viii. primary approximated message signal of STBC layer with $2 \times 1$ decoder

Step ix. V-BLAST layer depends on the initial approximated message signals of STBC.

Step x. Intrusion elimination.

Step xi. $\quad$ STBC with STBC $(2 M-2)$ decoder

Step xii. Stop.

\section{COMPUTATIONAL COMPLEXITIES OF PROPOSED QR DECOMPOSITION WITH CONVENTIONAL DECODER}

The dimension $2(M-1) \times 2 N$ of the channel transfer function $\mathrm{H}$ can be determined through complexity of traditional decoders such as zero force, minimum mean square and conventional QR decoder.

\section{a. ZF by means of Channel $\dot{H}$ :}

To compute the ZF equalizer $\mathrm{Y}$ from equation (7) is consists of following Steps.

Step i. $\quad 8(M-1)^{2} \times N$ multiplications and $4(M-1)^{2} \times(2 N-1)$ additions required for multiply $H^{H}$ with $H$. So total arithmetic operations required is $4(M-1)^{2} \times(4 N-1)$

Step ii. In this step Gaussian elimination matrix inversion of $H^{H} H$.Therefore the number of multifaceted calculation in this move is $2^{3}(M-1)^{3}$.

Step iii. Execute $\left(H^{H} H\right)^{-1}$ with. It needs $2^{3}(M-1)^{2} \times N$ multiplications and $2^{3}(M-1)^{2}-4(M-1) N$

Step iv. Additions. Therefore total arithmetic calculations in this step is $2^{4}(M-1)^{3} N-4(M-1) N$.

Step v. Calculate equalizer matrix $\mathrm{Y}$ multiplying with received vector .Therefore the total number of arithmetic operations in the ZF - VBLAST system is $2^{3}(M-1)^{3}+2^{2}(M-1)^{2} \times(8 N-1)+$ $2(M-1)(2 N-1)$.

\section{b. MMSE with Channel $\mathrm{H}$}

To calculate the MMSE equalizer filter matrix Y from equation (8) is consists of following Steps.

Step i. $\quad 8(M-1)^{2} \times N$ multiplications and $4(M-1)^{2} \times(2 N-1)$ additions required for multiply $H^{H}$ with $H$. So total arith matic operations required is $4(M-1)^{2} \times(4 N-1)$.

Step ii. performs addition of $H^{H} H$ with $\frac{1}{\operatorname{SNR}} \mathrm{I}$. Therefore the total arithmetic calculations in this step is $I 2(M-1)$.

Step iii. In this step Gaussian elimination matrix inversion of $\left(H^{H} H+\sigma^{2} I\right)$. Therefore arithmetic calculations in this step is $8(M-1)^{3}$.

Step iv. Execute $\left(H^{H} H+\sigma^{2} I\right)^{-1}$ with $H^{H} H$.

Step v. Calculate equalizer matrix Y multiplying with received vector. Therefore total number of arithmetic operations in MMSE-VBLAST system is $2^{3}(M-1)^{3}+2^{2}(M-1)^{2} \times(8 N-1)+2^{2}(M-1) N$.

\section{c. Traditional $\mathbf{Q R}$ decomposition by means of $\mathbf{H}$}

Step i. Now $\mathrm{QR}$ decomposition with $\mathrm{H}$ can be performed $Q H$ with received signal vector.Because the aspect of the channel transfer function is $2(M-1) \times 2 N$. Therefore total arithmetic operations of this method is $8 N(M-1)^{2}+\frac{8}{3}(1-M)(M-1)^{2}$. Thus total number of arithmetic calculations in $\mathrm{QR}$ decomposition is $8 N(M-1)^{2}+\frac{8}{3}(1-M)(M-1)^{2}+2(M-1)(4 N-1)$.

Step ii. It decodes departure symbols with towards the back replacement with cancellation and it needs $(2 \mathrm{M}-3) \times(\mathrm{M}-1)$ multiplications, $(\mathrm{M}-2) \times(2 \mathrm{M}-3)$ additions, $2(\mathrm{M}-1)-1$ subtractions and $2(\mathrm{M}-$ 1) divisions. Therefore the overall multifaceted sums calculations of straight $\mathrm{QR}$ decomposition is $8 N(M-1)^{3}+\frac{8}{3}(1-M)(M-1)^{2}+(2 M-3)^{2}+2(M-1)(4 N+1)-1$.

\section{d. Low complexities $Q R$ with $H$}

Step i. Apply QR decomposition [13-14] to the channel $\mathrm{H}$ and multiplication of $Q^{H}$ with $Y$. Now $N \times M$ is the dimension of the channel transfer function $\mathrm{H}$ therefore arithmetic calculation is $N M^{2} \frac{M^{3}}{3}$. After multiplication of $Q^{H}$ with $Y$ it require $M \times N$ multiplications and $(N-1) \times M$ additions'. Thus, total calculations in this step is is $N M^{2} \frac{M^{3}}{3}+.(2 N M-M)$. 
Step ii. In this first approximation signals of the STBC layer with2 $\times 2$ receiver. It requires 6 multiplications, 3 additions and 1 subtraction. Therefore arithmetic calculations in this step is 10 .

Step iii. In low complexity QR decomposition data symbols of V-BLAST layer of initial approximation sygnal contains $(M-1)(M-2)$ additions $2(M-2)$ subtractions $(M+1)(M-2)$ multiplications and $2(M-2)$ divisions. Thus the arithmetic calculations in this stage is $2(M-2)(M+2)$

Step iv. In this is for interference cancellation. Thus the number number of calculation in this step is $4 N(M-2)$.

Step v. Thus number of complex arithmetic operations in this step 2(4N-1). Ultimately, the total sums of LC-QR is $N M^{2}-\frac{M^{3}}{3}+(M+2)(2 N-1)+2\left(M^{2}-4\right)+4 N(M-1)+10$

Table1 shows comparisons of different decoders in which proposed LC-QR decoder provides less arithmetic operations than other mentioned decoders

Table 1. Comparison of Different Decoders

\begin{tabular}{cc}
\hline Decoders & Total complex arithmatic operations \\
\hline ZF with H & $8(M-1)^{3}+4(M-1)^{2} \times(8 N-1)+2(M-1)(2 N-1)$ \\
MMSE with $\mathrm{H}$ & $2^{3}(M-1)^{3}+2^{2}(M-1)^{2} \times(8 N-1)+(4 N M-4 N)$ \\
QR with $\mathrm{H}$ & $8 N(M-1)^{3}+\frac{8}{3}(1-M)(M-1)^{2}+(2 M-3)^{2}+2(M-1)(4 N+1)-1$ \\
Low complexity-QR with $\mathrm{H}$ & $N M^{2}-\frac{M^{3}}{3}+(M+2)(2 N-1)+2\left(M^{2}-4\right)+4 N(M-1)+1$ \\
\hline
\end{tabular}

\section{ERROR PROBABILITY IN V-BLAST SYSTEM WITH LOW COMPLEXITIES QR DECOMPOSITION}

The arriving signal in MIMO [15-17] communication system be able to be described since $r=$ $H X+n$ The channel order is $\mathrm{N} \times \mathrm{M}$ and $\mathrm{n}$ is nothing but AWGN having $\mathrm{N} \times 2$ matrices with variance $\sigma_{n}{ }^{2}$. The covariance matrix of $\mathrm{x}$ is $E\left[X X^{*}\right]=\sigma_{X}{ }^{2} I$. The signal to noise ratio (SNR) can be denoted as $\gamma=\frac{\sigma_{X}{ }^{2}}{\sigma_{n}{ }^{2}}$

In a simulation using matlab shown in Figure 10 which describes error probability of $4 \times 4 \mathrm{~V}$ BLASTZF,MMSE and QR decomposition detection. Comparision graph shows QR decomposition method provides better performance than traditonal ZF and MMSE counterpart. As far as low complexity QR method is concerned it provides better result than traditional QR,MMSE ,ZF and ZF-IS which is shown in Figure 11.

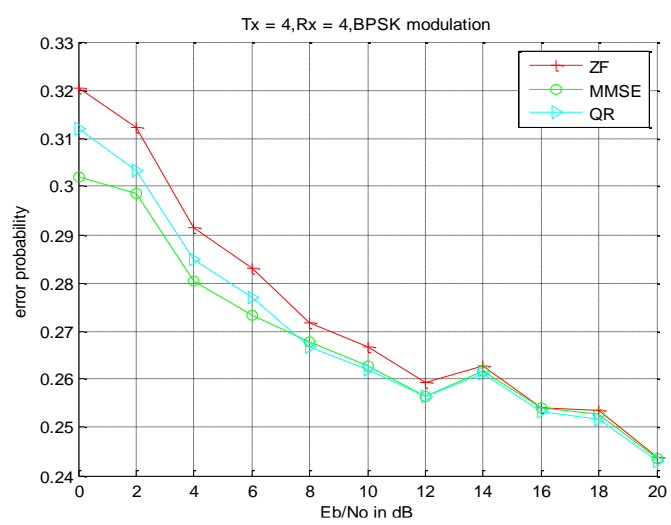

Figure 10. Error probability of $4 \times 4 \mathrm{~V}$-BLASTZF, MMSE and QR decomposition detection

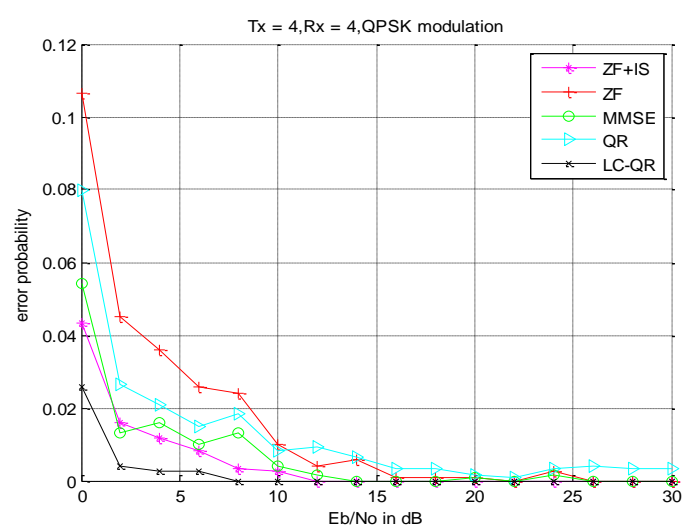

Figure 11. Error probability of proposed $4 \times 4 \mathrm{~V}-$ BLAST

\section{PROPOSED V-BLAST WITH VARIABLE ITERATIONS}

BER performances of V-BLAST variable iteration method can be shown in Figure 12. Table 2 shows iterations Vs SNR.As number of iteration increases, particularly 7th iteration yields low SNR correspondingly significantly improvement of BER as shown in Figure 12. The proposed algorithm can be applied to $4 \times 4$ MIMO-QPSK scheme. Begin with the first iteration procedure make sure if the estimated SNR gives to the accepted BER or not. If yes the rest of the symbols are linearly detected or else, the 
iterations carry on a waiting reaching the BER requirement.But the only drawback is that for low values of SNR it increases the computational complexity. We may use also another method to directly find out the number of iterations, from the SNR value.

Step i. Initializing(ordering)

Step ii. Number of iterations $\mathrm{Ki}=1$

Step iii. Nulling, slicing and cancellation

Step iv. If our targeted BER is achieved or $K_{i}=K_{\max }$ go to step-v else $K_{i}=K_{i}-1$ go to step-iii

Step v. Stop iterations and MMSE for rest.

\section{Direct method:}

Step i. Step:i Initializing(ordering)

Step ii. Step:ii Nulling, slicing and cancellation

Step iii. Step:iii Get SNR value from estimator

Step iv. Step:iv No of iterations $=\min \left(\frac{N_{t}}{2}, \max \left(\operatorname{round}\left(\frac{3}{4}(19-S N R)\right)\right)\right.$

Step v. Step:v MMSE linear detection for rest.

Step vi. Step:vi stop

Table 3 shows performance of complexity ratio using different algorithm in which the direct method gives $58 \%$ complexity ratio as compared with ordinary V-BLAST.

Table 2. Iterations Vs SNR

\begin{tabular}{ll}
\hline Number of iterations : & 766543211 \\
\hline SNR in dB: & 2.23 .74 .46 .27 .89111220 \\
\hline
\end{tabular}

Table 3. Complexity Ratio

\begin{tabular}{cc}
\hline Algorithm & Complexity ratio in $\%$ \\
\hline Ordinary v-blast & 100 \\
Feedback algorithm & 128 \\
Iterations at $K_{i}=K_{\text {imax }}$ & 56.76 \\
Direct method & 58 \\
\hline
\end{tabular}

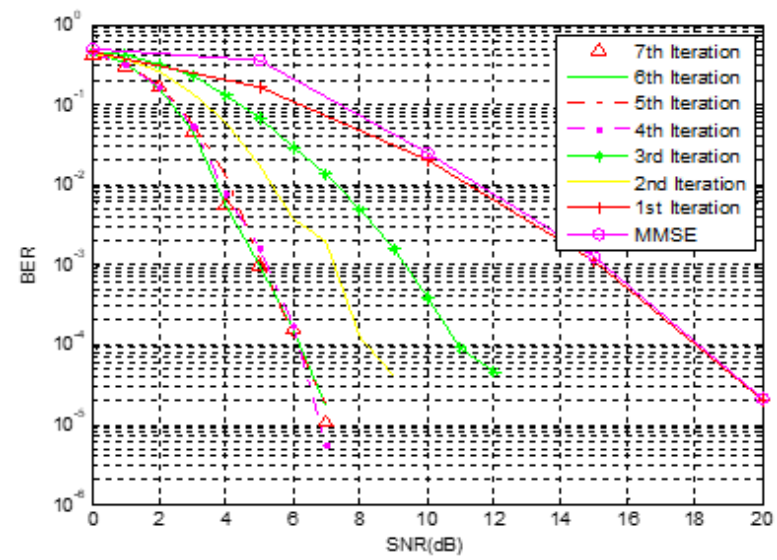

Figure 12. BER performances of V-BLAST variable iteration method

\section{CONCLUSION}

In this paper we have analyzed different equalization scheme along with VBLAST MIMO system.We have proposed two methods i.e low complexity QR decomposition and variable successive reduction method which is better than a contemporary VBLAST system by means of low BER and probability error. In low complexity QR decomposition method gives significantly reduced arithmetic complexities and variable successive reduction method we gain $52 \%$ complexity reduction without change in BER in a specified SNR. 


\section{REFERENCES}

[1] W. Yan, S. Sun, and Z. Lei, "A low complexity VBLAST OFDM detection algorithm for wireless LAN systems," IEEE Communications Letters, vol. 8, no. 6, pp. 374-376, Jun. 2004.

[2] Z. Luo, S. Liu, M. Zhao, and Y. Liu, "A Novel Fast Recursive MMSE-SIC Detection Algorithm for V-BLAST Systems," IEEETransactions on Wireless Communications, vol. 6, no. 6, pp. 2022-2025, Jun. 2007.

[3] Henuchul Le, Byeongsi Lee, and Inkyu Lee, "Iterative detection and decoding with an improved V-BLAST for MIMO-OFDM systems," IEEE Journal on Selected Areas in Communications, vol. 24, no. 3,pp. 504-513, Mar. 2006.

[4] T. Kim and S. C. Park, "Reduced complexity detection for V-BLAST systems from iteration cancelling," in Proc. 23rd International Technical Conference on Circuits/Systems, Computers and Communications, Shimonoseki, 2008, pp. 497-500.

[5] Sandhu S, Paulraj A (2000) Space-Time Block Codes: A Capacity Perspective. IEEE Commun Lett 4(12):384-386.

[6] S. M. Alamouti, "A simple transmit diversity technique for wireless communications," IEEE J. Select. Areas Comm., Vol.16, No.8, October 1998.

[7] G. J. Foschini, "Layered space-time architecture for wireless communication in a fading environment when using multi-elemen antennas", Bell Labs Technical Journal, 1996.

[8] Windpassinger, C., \& Fischer, R. F. (2003, March). Low-complexity near-maximum-likelihood detection and precoding for MIMO systems using lattice reduction. In Information Theory Workshop, 2003. Proceedings. 2003 IEEE (pp. 345-348). IEEE.

[9] Mao T, Motani M (2005) STBC-VBLAST for MIMO Wireless Communication Systems. Proc IEEE ICC (ICC 2005)4:2266-2270.

[10] Tarokh V, Jafarkhani H, Calderbank AR (1999) Space-Time Block Codes from Orthogonal Designs. IEEE Trans Inf Theory 45(5): 1456-1467.

[11] Wang H, Xia XG (2003) Upper Bounds of Rates of Complex Orthogonal Space-Time Block Codes. IEEE Trans Inf Theory 49(10):2788-2796.

[12] ongoria-Gandara O, Sanchez-Hernandez A, Cortez J, Bazdresch M, Parra-Michel R (2007) Linear Dispersion Codes Generation from Hybrid STBC-VBLAST Architectures. In: Proceedings of 4th International Conference Electrical and Electronics Engineering (ICEEE 2007) 142-145.

[13] Gorokhov A, Gore DA, Paulraj AJ (2003) Receive Antenna Selection for MIMO Spatial Multiplexing: Theory and Mechanisms. IEEE Trans Signal Process 51(11):2796-2807.

[14] Bohnke, R., Wubben, D., Kuhn, V., \& Kammeyer, K. D. (2003, December). Reduced complexity MMSE detection for BLAST architectures. In Global Telecommunications Conference, 2003. GLOBECOM'03. IEEE (Vol. 4, pp. 2258-2262). IEEE.

[15] Henuchul Le, Byeongsi Lee, and Inkyu Lee, "Iterative detection and decoding with an improved V-BLAST for MIMO-OFDM systems," IEEE Journal on Selected Areas in Communications, vol. 24, no. 3,pp. 504-513, Mar. 2006.

[16] Zheng L, Tse DNC (2003) Diversity and Multiplexing: A Fundamental Trade off in Multiple Antenna Channels. IEEE Trans Inf Theory 49(5):1073-1096

[17] Sandeep G, Ravi-Teja C, Kalyana-Krishnan G, Redy VU (2007) Low Complexity Decoders for Combined Space Time Block Coding and V-BLAST. In: Proceedings of IEEE Wireless Communications and Networking Conference (WCNC 2007) 582-587. 